\title{
Cobertura y calidad del registro de defunciones perinatales ocurridas en instituciones de salud del Distrito Federal
}

\author{
José L. Bobadilla* \\ Prudencia Cerón $^{\star *}$ e Irma Coria ${ }^{* *}$
}

\begin{abstract}
En este artículo se presentan los resultados de la primera evaluación directa de la cobertura del registro de muertes perinatales en el Distrito Federal, tomando como base la información sobre 574 defunciones ocurridas en 23 hospitales del Distrito Federal en un trimestre de 1984; ellas fueron captadas en una investigación sobre la calidad de la atención médica y su efecto sobre la mortalidad perinatal. Aquí se señalan y discuten los determinantes del subregistro y la calidad de la información de los certificados de defunción perinatal. De acuerdo con los datos analizados, se puede concluir que el nivel de subregistro encontrado para el Distrito Federal es muy alto, sobre todo si se considera la utilización de las tasas de mortalidad para la planificación de los servicios de salud perinatal. Se sugiere la posibilidad de establecer un sistema de registro hospitalario tanto de defunciones como de nacimientos, así como la necesidad de establecer normas para la captación de muertes fetales y la urgencia de unificar criterios con respecto a la definición de "nacido vivo".
\end{abstract}

Introducción y antecedentes

Se estima que en nuestro país las estadísticas vitales captan $90 \%$ de las defunciones (Cordero, 1968). Éste es el mínimo recomendado por acuerdos internacionales para considerarlas como completas; sin embargo, el subregistro no se distribuye homogéneamente entre todos los grupos de edad, observándose una mayor omisión de muertes de menores de un año (Ordorica y otros, 1975). Algunos estudios han intentado medir a partir de diversos métodos el grado de omisión del registro de muertes infantiles (Ordorica y otros, 1975; Aguirre y Camposortega, 1980; Suárez, 1981). Todos coinciden en que las tasas de mortalidad infantil elaboradas con datos provenientes del Registro Civil son inferiores a las obtenidas por otros métodos, calculando que la subestimación oscila entre 25 y 45 por ciento para 1940, entre 10 y 35 para 1960 y entre 0 y 30 para 1970 (Aguirre y Camposortega, 1980).

Por otro lado, también se observan diferencias notables en las cifras de mortalidad infantil por entidades federativas (Cordero, 1968; Ordorica y otros, 1975; Suárez, 1981). La tasa de mortalidad infantil es un indicador Salud.

* Investigador titular del Centro de Investigaciones en Salud Pública de la Secretaría de de Salud.

Investigador asociado del Centro de Investigaciones en Salud Pública de la Secretaría 
sensible del desarrollo socioeconómico y de las condiciones de vida de una población. De esta manera, Guerrero, Oaxaca, Chiapas e Hidalgo, entre otras entidades atrasadas y con una economía comparativamente poco desarrollada, muestran paradójicamente tasas de mortalidad más bajas que las de estados con mayor índice de desarrollo socioeconómico (Suárez, 1981). Esto confirma el hecho de que existe una omisión importante de muertes infantiles; en contraste, hay entidades como el Distrito Federal que se considera tienen un registro de defunciones infantiles mucho más completo (Cordero, 1968; Suárez, 1981; Ordorica y otros, 1978).

Otra evidencia de la subestimación de la mortalidad infantil aparece en las estadísticas vitales; en la distribución de las muertes por edad al morir, es mayor el número de muertes reportadas en el segundo día de vida que en el primero, hecho poco factible, ya que la probabilidad de morir en el primer día es más alta que en los subsiguientes (Ordorica y otros, 1975).

En México se han efectuado algunos estudios para evaluar directamente la magnitud del problema del subregistro de muertes, utilizando principalmente censos de nacimientos y defunciones, encuestas domiciliarias y búsqueda manual de los diversos documentos de defunción (Varela y Narro, 1981; Canales y otros 1980; Ordorica y otros, 1978). Los hallazgos muestran un subregistro de $84.6 \%$ para muertes infantiles en Mazatepec (Ordorica y otros 1978), y de 83\% para mortalidad general en Ixtlilco el Grande (Canales y otros, 1980), ambos municipios del estado de Morelos. No existe evidencia de que las defunciones no registradas en estos municipios no hayan sido inscritas en otros, pues a pesar de que sólo $11 \%$ de las oficialías del Registro Civil están autorizadas para captar actos fuera de su jurisdicción (Secretaría de Gobernación, 1982: 20), es un fenómeno común la inscripción de muertes que no corresponden a la jurisdicción de las oficialías, siendo más frecuente que se registren en zonas donde hay una concentración de centros hospitalarios, esto es, en el lugar donde ocurre el mayor número de muertes.

Además de las omisiones en el registro, existen deficiencias en la información captada tanto en actas y certificados de defunción, como en las hojas mensuales de estadística. Un ejemplo se observa en los datos sobre la residencia habitual y el sexo, que muestran altas cifras de no concordancia. Otro problema se deriva de la relativa baja proporción de certificación médica. En 1974, 18\% de las muertes infantiles no fueron certificadas por un médico (Natali, 1978: 447). Además, es frecuente que las personas fallecidas no hayan sido atendidas durante su última enfermedad por el médico certificante, lo que pone en duda la calidad de la información sobre las causas de la muerte.

La cobertura del registro de muertes perinatales, que incluye defunciones fetales tardías (nacidos muertos con 28 o más semanas de gestación) y neonatales tempranas (nacidos vivos que fallecen en los primeros 
siete días de vida), no ha sido evaluada en México. Es de esperar, por los resultados de otras investigaciones, que el subregistro de muertes perinatales sea mayor que el del resto de las defunciones infantiles. La discrepancia entre la definición de "nacido vivo" del artículo 337 del Código civil del Distrito Federal, en la que se señala que el recién nacido debe vivir más de 24 horas para ser considerado como vivo, y la propuesta en 1978 por la Organización Mundial de la Salud, en la que se define al nacido vivo como aquel ser que, desprendido del seno materno, "da señales de vida tales como latidos cardiacos, pulsaciones del cordón umbilical, movimientos de los músculos de contracción voluntaria. . ., etc.", sugiere que posiblemente exista transferencia de defunciones neonatales tempranas a fetales tardías (Natali, 1978). Un fenómeno observado por algunos autores es la relación inversa entre el peso al nacer y el subregistro de muertes perinatales. Un estudio realizado en Georgia, E.U., informa de un subregistro de $21 \%$ para los recién nacidos que contaban con un peso al nacer menor de $1500 \mathrm{~g}$ (McCarthy y otros, 1977). Asimismo, en un estudio realizado en 1970 en Santiago de Chile, se encontró que $56.1 \%$ de las defunciones neonatales no habían sido registradas, siendo mayor la omisión conforme descendía el peso de los neonatos hasta llegar a $80 \%$ de subregistro en los menores de $1500 \mathrm{~g}$ (Legarreta y otros, 1973).

Ante el desconocimiento de la magnitud del subregistro de muertes perinatales en las estadísticas vitales, se decidió efectuar un estudio que permitiera estimar el subregistro, analizar sus determinantes y la calidad de la información de los certificados de un grupo de defunciones perinatales.

Material y método

Al ocurrir una defunción en el Distrito Federal, debe inscribirse en el Registro Civil como requisito para la inhumación. Después de ese registro, el oficial del Registro Civil debe enviar una copia del certificado de defunción a la Secretaría de Salud. Esto se hace mensualmente, remitiendo las copias de los certificados de defunción a la jurisdicción sanitaria que corresponde. Hasta antes de 1985, de la jurisdicción eran enviadas mensualmente a la Dirección General de los Servicios de Salud Pública en el Distrito Federal (DGSSPDF).

En la DGSSPDF se llevó a cabo una búsqueda manual de certificados de defunción de 574 muertes perinatales: 260 fetales tardías y 314 neonatales tempranas. Todas ellas fueron identificadas durante el censo de nacimientos de una investigación sobre la atención médica y su efecto sobre la mortalidad perinatal, realizada del 21 de mayo al 26 de agosto de 1984 en 23 hospitales de la zona metropolitana de la ciudad de México. Los hospitales eran representativos de las tres instituciones de salud más importan- 
tes: el Instituto Mexicano del Seguro Social (IMss), el Instituto de Seguridad Social al Servicio de los Trabajadores del Estado (ISSSTE) y la Secretaría de Salubridad y Asistencia (ssA), incluyendo además los cuatro hospitales privados más grandes. La metodología y los resultados obtenidos de esa investigación han sido descritos en Bobadilla (1985).

Durante la investigación realizada en 1984, a las madres con defunciones perinatales se les aplicó un cuestionario en el que se recabó información socioeconómica y de algunas características biológicas de la madre, así como información sobre la atención médica recibida. En la investigación se captaron 976 muertes perinatales; sin embargo, se decidió estudiar sólo aquellas muertes de recién nacidos cuyas madres residían en el Distrito Federal.

En la DGSSPDF se verificó, con la colaboración de cinco pasantes de enfermería, que estuvieran concentrados todos los certificados de muertes ocurridas durante 1984 en el Distrito Federal; luego se seleccionaron documentos con las siguientes características: certificados de defunción fetal que indicaran una edad gestacional de 26 o más semanas; certificados de defunción de productos nacidos vivos que estipularan que la muerte había ocurrido en los primeros 15 días de vida, y certificados de defunción tanto fetal como neonatal que no consignaran las semanas de gestación o la edad al morir, respectivamente.

Se definieron las defunciones fetales tardías como aquéllas con 28 o más semanas de gestación, y las neonatales tempranas como aquéllas ocurridas en los primeros siete días de vida; no obstante, se dio un amplio margen de error para estos datos en la selección de los certificados, tratando de preveer posibles errores en la declaración de la edad gestacional y de la edad al morir.

Antes de separar los certificados de muertes perinatales, se elaboró una libreta con las defunciones del estudio de mortalidad perinatal, con los siguientes datos del cuestionario aplicado a la madre: nombre y apellidos de la madre, lugar de residencia habitual de la misma, sexo del recién nacido, fecha de nacimiento, fecha de defunción, edad al morir y/o edad gestacional. Posteriormente, se ordenaron por hospital donde ocurrió la muerte y se clasificaron alfabéticamente por apellido paterno de la madre. Se procedió entonces a confrontar manualmente esta información con cada certificado de defunción que llenaba las características mencionadas. De esta manera se seleccionaron todos los certificados coincidentes.

La información de los certificados de defunción localizados se transcribió a una forma precodificada y se procesó electrónicamente. En cada etapa se realizó una doble verificación del trabajo, que consistió en revisión y supervisión de las actividades por otros miembros del equipo. 


\section{Resultados}

\section{Cobertura del registro}

De las 574 defunciones perinatales estudiadas, $126(22 \%)$ no fueron inscritas en el Registro Civil. Por tipo especifico de defunción, se encontro $24 \%$ de subregistro para muertes fetales y $20 \%$ para las neonatales (véase el cuadro 1).

En el cuadro 2 se presentan los porcentajes de registro de defunciones perinatales por delegación política de residencia materna. El subregistro fue de 20 a $30 \%$ en la mayoría de las delegaciones, aunque en algunas fue muy alto, como en Magdalena Contreras, donde fue de $50 \%$. Esto contrasta con delegaciones como Miguel Hidalgo (con $11 \%$ de subregistro) y Gustavo A. Madero con el subregistro más bajo encontrado (4 por ciento).

En el cuadro 3 observamos la distribución de defunciones por delegación de residencia materna y la distribución por delegación donde realmente se inscribieron. Por un lado se puede apreciar que en cinco delegaciones (Cuajimalpa, Iztacalco, Milpa Alta, Tláhuac y Xochimilco), no se inscribió ninguna muerte perinatal. Por otro lado, en Álvaro Obregón se inscribieron 23 defunciones, pero sólo 15 correspondían realmente a esa delegación. Una situación parecida se observa en la Cuauhtémoc, donde se registraron 161 defunciones, aunque sólo 40 pertenecían a la misma. En las restantes delegaciones se observa una tendencia a inscribir menos defunciones perinatales de las que realmente ocurrieron.

El cuadro 4 nos muestra la relación entre el peso al nacer y el registro de defunciones. Es notable que el porcentaje más alto de subregistro se presenta entre el grupo de menores de $1500 \mathrm{~g}$ con $27.5 \%$, disminuyendo conforme aumenta el peso. No obstante, hay un incremento del subregistro al llegar al grupo con peso más alto ( $300 \mathrm{~g}$ y más), con 22.4 por ciento.

En el cuadro 5 se presenta un resultado similar al anterior. En este caso, la baja edad gestacional de las defunciones perinatales tiene relación con un mayor subregistro, siendo de $41.3 \%$ entre las semanas 28 y 31 . Sin embargo, en los productos mayores de 43 semanas de gestación también

CUADRO 1

Defunciones perinatales registradas y no registrades según tipo de muerte

\begin{tabular}{llcccc}
\hline $\begin{array}{l}\text { Tipo de } \\
\text { defunción }\end{array}$ & Registradas & $\%$ & No registradas & $\%$ & Total \\
\hline Fetales & 198 & 75.15 & 62 & 23.84 & 260 \\
Neonatales & 250 & 79.61 & 64 & 20.38 & 314 \\
\hline Total & 448 & 78.04 & 126 & 21.95 & 574 \\
\hline
\end{tabular}




\section{CUADRO 2}

Cobertura del registro de defunciones perinatales según delegación de residencia materna

\begin{tabular}{lcccrr}
\hline $\begin{array}{c}\text { Delegación de } \\
\text { residencia }\end{array}$ & $\begin{array}{c}\text { Número de } \\
\text { registradas }\end{array}$ & \multicolumn{1}{c}{$\%$} & $\begin{array}{c}\text { Número de } \\
\text { no registradas }\end{array}$ & $\%$ & Total \\
\hline Alvaro Obregón & 15 & 60.0 & 10 & 40.0 & 25 \\
Azcapotzalco & 34 & 83.0 & 7 & 17.0 & 41 \\
Benito Juárez & 22 & 78.5 & 6 & 21.4 & 28 \\
Coyoacán & 22 & 84.6 & 4 & 15.3 & 26 \\
Cuajimalpa & 5 & 71.4 & 2 & 28.5 & 7 \\
Cuauhtémoc & 40 & 74.0 & 14 & 25.9 & 54 \\
Gustavo A. Madero & 100 & 96.1 & 4 & 3.8 & 104 \\
Iztacalco & 26 & 81.2 & 6 & 18.7 & 32 \\
Iztapalapa & 70 & 76.0 & 22 & 23.9 & 92 \\
Magdalena Contreras & 2 & 50.0 & 2 & 50.0 & 4 \\
Miguel Hidalgo & 24 & 88.8 & 3 & 11.1 & 27 \\
Milpa Alta & 2. & 66.6 & 1 & 33.3 & 3 \\
Tláhuac & 4 & 66.6 & 2 & 33.3 & 6 \\
Tlalpan & 25 & 73.5 & 9 & 26.4 & 34 \\
Venustiano Carranza & 38 & 79.1 & 10 & 20.8 & 48 \\
Xochimilco & 19 & $\mathbf{8 2 . 6}$ & 4 & 17.3 & 23 \\
\hline Total & 448 & 78.0 & 126 & 22.0 & $\mathbf{5 7 4}$ \\
\hline
\end{tabular}

se observa un alto porcentaje de subregistro (27.2 por ciento).

Dada la importancia tanto del peso al nacer como de la edad gestacional en relación a la cobertura, se efectuó el análisis del registro por peso al nacer, controlado por edad gestacional. En el cuadro 6 se muestra que entre los recién nacidos con 40 a 43 semanas de gestación, hay una relación inversa entre el peso al nacer y el subregistro, pues los que pesaron menos de $1500 \mathrm{~g}$ presentan un subregistro del orden de $80 \%$, cifra que disminuye a $29 \%$ en los que pesaron entre 1500 y $2499 \mathrm{~g}$ y a $12 \%$ en los de entre 2500 y $3499 \mathrm{~g}$. Estos hallazgos son estadísticamente significativos $(p<.005)$.

En relación con la institución donde tuvieron lugar las defunciones, el cuadro 7 señala que el mayor subregistro se dio en la asistencia pública con $28.5 \%$; el issSTE tuvo $26.5 \%$, en contraste con el iMSS, que mostró un porcentaje mucho más bajo, de $11.6 \%$. Los hospitales privados estudiados no presentaron subregistro.

El estado civil de la madre mostró estar asociado con el subregistro. Para las madres viudas, el subregistro fue de $\mathbf{4 0} \%$, para las separadas de $38 \%$ y para las solteras de $31 \%$. Entre las mujeres casadas y en unión libre el subregistro fue de 20 y $25 \%$, respectivamente.

Otras características de la madre con altos porcentajes de subregistro fueron: edad materna menor de 18 años, $30 \%$; ningún año de escolaridad, 
CUADRO 3

Distribución de defunciones perinatales por lugar de residencia y por lugar de registro

\begin{tabular}{|c|c|c|c|c|c|}
\hline \multirow{3}{*}{$\begin{array}{c}\text { Delegación } \\
\text { política }\end{array}$} & \multirow{3}{*}{$\begin{array}{l}\text { Defunciones } \\
\text { perinatales } \\
\text { ocurridas }\end{array}$} & \multicolumn{4}{|c|}{ Defunciones perinatales registradas } \\
\hline & & \multicolumn{2}{|c|}{$\begin{array}{c}\text { A } \\
\begin{array}{l}\text { Según lugar de } \\
\text { residencia }\end{array}\end{array}$} & \multicolumn{2}{|c|}{$\begin{array}{c}\text { B } \\
\begin{array}{c}\text { Según lugar de } \\
\text { registro }\end{array}\end{array}$} \\
\hline & & Número & $\%$ & Número & $\%$ de $\mathrm{A}$ \\
\hline Álvaro Obregón & 25 & 15 & & 23 & 153 \\
\hline Azcapotzalco & 41 & 34 & & 25 & 74 \\
\hline Benito Juárez & 28 & 22 & & 19 & 86 \\
\hline Coyoacán & 26 & 22 & & 5 & 23 \\
\hline Cuajimalpa & 7 & 5 & & - & $\mathbf{0}$ \\
\hline Cuauhtémoc & 54 & 40 & & 161 & 403 \\
\hline Gustavo A. Madero & 104 & 100 & & 91 & 91 \\
\hline Iztacalco & 32 & 26 & & - & 0 \\
\hline Iztapalapa & 92 & 70 & & 30 & 43 \\
\hline Magdalena Contreras & 4 & 2 & & 1 & 50 \\
\hline Miguel Hidalgo & 27 & 24 & & 14 & 58 \\
\hline Milpa Alta & 3 & 2 & & - & $\mathbf{0}$ \\
\hline Tláhuac & 6 & 4 & & - & o \\
\hline Tlalpan & 34 & 25 & & 25 & 100 \\
\hline Venustiano Carranza & 48 & 38 & & 34 & 89 \\
\hline Xochimilco & 23 & 19 & & - & 0 \\
\hline Se ignora & - & - & & 20 & 一 \\
\hline Total & 574 & 448 & & 448 & 100 \\
\hline
\end{tabular}

$27 \%$; entre cinco y siete hijos, $27 \%$, y ocupación de trabajadoras directas en el proceso de producción industrial, 33.3\% (Secretaría de Programación y Presupuesto, 1980); las trabajadoras en servicios domésticos remunerados mostraron 33.3 por ciento.

Del total de certificados localizados, ocho defunciones neonatales tenían uno de muerte fetal. La transferencia fue de $2 \%$ con respecto al total

\section{CUADRO 4}

Cobertura del registro de defunciones perinatales según peso al nacer en gramos

\begin{tabular}{lccccc}
\hline Peso al nacer & Registradas & $\%$ & No registradas & $\%$ & Total \\
\hline $500-1499$ & 145 & 72.5 & 55 & 27.5 & 200 \\
$1500-2499$ & 153 & 80.1 & 38 & 19.3 & 191 \\
$2500-3499$ & 102 & 85.8 & 17 & 14.2 & 119 \\
3500 o más & 38 & 77.6 & 11 & 22.4 & 49 \\
Se ignora & 10 & 66.7 & 5 & 33.3 & 15 \\
\hline Total & 448 & 78 & 126 & 22 & 574 \\
\hline
\end{tabular}


CUADRO 5

Cobertura del registro de defunciones perinatales según edad gestacional

\begin{tabular}{lccccr}
\hline $\begin{array}{c}\text { Edad } \\
\text { gestacional }\end{array}$ & $\begin{array}{c}\text { Número de } \\
\text { registradas }\end{array}$ & $\%$ & $\begin{array}{c}\text { Número de } \\
\text { no registradas }\end{array}$ & $\%$ & Total \\
\hline $28-31$ & 27 & 56.6 & 19 & 41.3 & 46 \\
$32-35$ & 38 & 86.3 & 6 & 13.6 & 44 \\
$36-39$ & 70 & 73.6 & 25 & 26.3 & 95 \\
$40-42$ & 50 & 81.9 & 11 & 18.0 & 61 \\
43 & 8 & 72.7 & 3 & 27.2 & 11 \\
Se ignora & 5 & 100.0 & - & 0.0 & 5 \\
\hline Total & 198 & 78.0 & 64 & 22.0 & 262 \\
\hline
\end{tabular}

CUADRO 6

Cobertura del registro de defunciones perinatales en niños con 40 a 43 semanas de gestación, según peso al nacer

\begin{tabular}{lccccr}
\hline Peso al nacer & $\begin{array}{l}\text { Número de } \\
\text { registradas }\end{array}$ & $\%$ & $\begin{array}{c}\text { Número de } \\
\text { no registradas }\end{array}$ & $\%$ & Total \\
\hline $\mathbf{5 0 0 - 1 4 9 9}$ & 1 & 20.0 & 4 & $\mathbf{8 0 . 0}$ & 5 \\
$1500-2499$ & 10 & 71.4 & 4 & 28.6 & 14 \\
$2500-3499$ & 24 & 88.0 & 3 & 11.1 & 27 \\
3500 o más & 20 & 87.0 & 3 & 13.0 & 23 \\
Se ignora & 3 & 100.0 & - & - & 3 \\
\hline Total & 58 & $\mathbf{8 0 . 5}$ & 14 & 19.4 & 72 \\
\hline
\end{tabular}

CUADRO 7

Cobertura del registro de defunciones perinatales según institución de nacimiento

\begin{tabular}{lccccr}
\hline $\begin{array}{c}\text { Institución de } \\
\text { nacimiento }\end{array}$ & $\begin{array}{c}\text { Número de } \\
\text { registradas }\end{array}$ & $\%$ & $\begin{array}{c}\text { Número de } \\
\text { no registradas }\end{array}$ & $\%$ & Total \\
\hline Asistencia pública & 168 & 71.4 & 67 & 28.5 & 235 \\
Seguridad social 1 & 105 & 73.4 & 38 & 26.5 & 143 \\
Seguridad social 2 & 160 & $\mathbf{8 8 . 3}$ & 21 & 11.6 & 181 \\
Sector privado & 15 & 100.0 & - & - & 15 \\
\hline Total & 448 & $\mathbf{7 8 . 0}$ & 126 & 22.0 & 574 \\
\hline
\end{tabular}


CUADRO 8

Defunciones perinatales registradas y atención médica

\begin{tabular}{|c|c|c|c|c|c|c|}
\hline $\begin{array}{l}\text { EI médico que } \\
\text { certifica, } \\
\text { atendió el parto } \\
\text { y/o al fallecido }\end{array}$ & $\begin{array}{l}\text { Defunciones } \\
\text { fetales }\end{array}$ & $\%$ & $\begin{array}{c}\text { Defunciones } \\
\text { neonatales }\end{array}$ & $\%$ & \multicolumn{2}{|c|}{ Total } \\
\hline$\overline{\text { Sí }}$ & 48 & 24.2 & 91 & 36.4 & 139 & 31.0 \\
\hline No & 144 & 72.7 & 154 & 61.6 & 298 & 66.5 \\
\hline Se ignora & 6 & 3 & 5 & 2 & 11 & 2.4 \\
\hline Total & 198 & 100.0 & 250 & 100.0 & 448 & 100.0 \\
\hline
\end{tabular}

de muertes perinatales. El más alto porcentaje de transferencia se observó en el ISSSTE con $4 \%$ en relación con todas las defunciones perinatales ocurridas en esa institución. Cabe señalar que $75 \%$ de las transferencias observadas en el iSSSTE correspondieron a un solo hospital.

\section{Calidad de los datos}

Todos los certificados de defunción, tanto de muertes fetales como neonatales, contaban con certificación médica; no obstante, en el caso de las muertes fetales sólo en $\mathbf{2 4} \%$ de los casos el médico certificante fue el mismo que atendió el parto (véase el cuadro 8), en tanto que en el caso de las muertes neonatales sólo en $36 \%$ de los casos, el médico que certificó fue el mismo que atendió al neonato.

Para evaluar la concordancia de los datos, se compararon los consignados en los certificados de defunción con los recabados durante la investigación de mortalidad perinatal; se tomaron como buenos los datos de la investigación y el nivel exigido de precisión de los certificados no permitió la más mínima variación. Los resultados aparecen en el cuadro 9 . La discordancia más alta se observó en la edad gestacional: $44.6 \%$ no coincidian; también la ocupación del padre tuvo un alto porcentaje de nó concordancia $(31.8 \%)$, siguiéndole la hora de defunción de los neonatos $(19.2 \%)$, la hora de nacimiento de las muertes fetales (19.1\%) y el día de nacimiento $(17.1 \%)$. En lo que respecta a la delegación de residencia, no coincidieron $14.7 \%$ de los certificados; hay que destacar que la omisión de este dato fue de 24.5 por ciento.

\section{Discusión}

La muestra de muertes perinatales seleccionada en la investigación sobre la atención médica y su efecto sobre la mortalidad perinatal es representativa de las muertes perinatales ocurridas en las tres principales institucio- 
CUADRO 9

Evaluación de la precisión de los datos

\begin{tabular}{lccrrr}
\hline Variable & $\begin{array}{c}\text { Certificados } \\
\text { comparados }\end{array}$ & $\begin{array}{c}\text { No } \\
\text { coincidencia }\end{array}$ & \multicolumn{1}{c}{$\%$} & Omisiones & \multicolumn{1}{c}{$\%$} \\
\hline Sexo & 448 & 11 & 2.4 & 2 & 0.4 \\
Delegación de residencia & 448 & 66 & 14.7 & 110 & 24.5 \\
Entidad federativa de & & & & & \\
residencia & 448 & 18 & 4.0 & 100 & 22.3 \\
Edad gestacional & 204 & 91 & 44.6 & 3 & 1.4 \\
Fecha de nacimiento (día) & 204 & 35 & 17.1 & 4 & 1.9 \\
Fecha de nacimiento (mes) & 204 & 3 & 1.4 & 4 & 1.9 \\
Hora de nacimiento & 204 & 39 & 19.1 & 3 & 1.4 \\
Edad de la madre & 204 & 38 & 18.6 & 1 & 0.4 \\
Ocupación de la madre & 204 & 41 & 20.0 & 2 & 0.9 \\
Ocupación del padre & 204 & 65 & 31.8 & 32 & 15.6 \\
Fecha de defunción (día) & 244 & 33 & 13.5 & 1 & 0.4 \\
Fecha de defunción (mes) & 244 & 1 & 0.4 & 1 & 0.4 \\
Hora de defunción & 244 & 47 & 19.2 & 1 & 0.4 \\
Edad al morir & 244 & 76 & 31.1 & 5 & 2.0 \\
\hline
\end{tabular}

nes de salud en México: SSA, iMSS e ISSSTE. No se incluyen defunciones de recién nacidos ocurridas en el hogar, ni tampoco aquéllas ocurridas en hospitales privados con menos de dos mil nacimientos atendidos por año, así como las de recién nacidos que tuvieron lugar en instituciones diferentes a las estudiadas. Por lo tanto, es difícil generalizar los resultados obtenidos al resto de los nacimientos ocurridos en el Distrito Federal.

De acuerdo con la información proveniente de las estadísticas hospitalarias de la Dirección General de Estadística, en las instituciones mencionadas se atienden aproximadamente $75 \%$ de los nacimientos que ocurren en el Distrito Federal, lo que pone de manifiesto la importancia de los resultados.

Este trabajo de investigación ha producido la primera estimación directa del subregistro de defunciones perinatales en México. El $22 \%$ de subregistro encontrado contrasta desfavorablemente con la ausencia de subregistro observado en Cuba (Rojas y Sánchez, 1977), así como con el insignificante nivel encontrado en Washington, E.U., de $2.6 \%$ (Frost, 1982). Sin embargo, el nivel de subregistro determinado por este trabajo es menor al observado en otros estudios sobre México como los de Ordorica y otros (1978) y Canales y otros (1980), ambos efectuados en zonas rurales de México donde las cifras son del orden de 84.6 y $83 \%$, respectivamente, tanto para mortalidad infantil como para mortalidad general.

Hasta ahora se consideraba al Distrito Federal como una de las entidades con estadísticas vitales completas. Este trabajo sugiere, sin embargo, que el problema del subregistro no está resuelto en el Distrito Federal, al menos en lo que respecta a defunciones perinatales. 
El nivel de subregistro encontrado para el Distrito Federal es muy alto si se considera el uso de las tasas de mortalidad en la planeación de los servicios de salud perinatal. Esta situación se agrava aún más por el flujo de inscripción de muertes que se observa por delegaciones. El análisis de datos sobre mortalidad perinatal o infantil por delegaciones está entonces contraindicado mientras se utilicen datos crudos de las estadísticas vitales.

El flujo de la inscripción de defunciones no sólo se da entre delegaciones políticas, sino también entre estados. En efecto, hay evidencia de que una cantidad considerable de defunciones ocurridas en el estado de México son registradas en el Distrito Federal (Partida, 1982). Esto sugiere que las tasas de mortalidad perinatal para el Distrito Federal se encuentran compensadas por el sobrerregistro antes aludido. No están todas las que son y no son todas las que están.

Es muy difícil, con base en los resultados de este trabajo, especular sobre la situación de la cobertura del registro en otras entidades federativas. Por una parte, el mayor desarrollo socioeconómico que presenta el Distrito Federal en relación con el resto parecería indicar que en otros estados o ciudades el subregistro puede ser aún mayor. Hay, sin embargo, dos factores que sugieren que otras ciudades o estados tienen una cobertura mayor que el Distrito Federal de defunciones infantiles.

En primer lugar, en el Distrito Federal había, en 1980, 275803 habitantes por oficialía del Registro Civil, una cifra más alta que la de los 31 estados. Así, la accesibilidad al registro civil es significativamente menor en la capital.

En segundo término, la cobertura de la atención hospitalaria es mayor en ciudades como Monterrey, donde probablemente la cobertura del registro también es mayor.

Cabe señalar que la explicación dada por Partida (1982) para el flujo de inscripción de muertes del estado de México al Distrito Federal, es la misma que se encontró en este estudio para entender el flujo entre delegaciones. Se trata de la búsqueda de servicios de atención médica de nivel hospitalario. En efecto, en la delegación Cuauhtémoc en donde se concentró más de $80 \%$ de las defunciones inscritas fuera del lugar de residencia, se ubican seis de los 23 hospitales incluidos en el estudio. Además, esos seis hospitales eran de los más grandes de la ciudad de México.

Este flujo del registro entre estados y delegaciones no representaría mayor problema en el manejo estadístico de información por parte de la Dirección General de Estadística, si ésta se procesara según lugar de residencia habitual. Sin embargo, la omisión de este dato en $24.5 \%$ de los certificados presupone que las defunciones se procesan según la delegación donde se inscribió la muerte, lo que ocasiona una sobrestimación de la mortalidad perinatal en delegaciones como Cuauhtémoc y Álvaro Obregón, y una subestimación en el resto.

Conviene reflexionar sobre posibles causas del subregistro no necesa- 
riamente ligadas a las características del sistema del registro civil. El marco legal que ofrece el registro de hechos vitales como el divorcio o el nacimiento de un niño, probablemente sea menos claro cuando se trata de defunciones y matrimonios, que se presentan junto con uniones libres y subregistro de defunciones. Las defunciones ocurridas entre recién nacidos o antes del nacimiento (fetales) tienen seguramente un valor distinto cuando se les compara con el resto de defunciones. Resulta lógico que el valor sea menor si se toma en consideración la clásica subenumeración censal de menores de un año.

El Registro Civil en México se caracteriza por su pasividad; asume que la población está lo suficientemente educada como para hacer uso de sus servicios. Sería conveniente, sin embargo, estudiar los factores sociales y culturales que, bajo condiciones de accesibilidad, determinan la decisión de no registrar una muerte perinatal.

Las muertes perinatales estudiadas aquí constituyen, como ya se dijo, una muestra sesgada por haber ocurrido en hospitales. El sesgo con respecto a la probabilidad de registro es positivo. Es entonces sorprendente que una proporción tan alta de muertes no se registre. ¿Por qué, si se sabe que en grandes ciudades la mayor parte de las muertes ocurren en hospitales, no se han facilitado los procedimientos para su registro automático en los hospitales mismos? La respuesta quizá se derive de la postura pasiva del Registro Civil.

Si tomamos en cuenta que una alta proporción de los partos en el Distrito Federal tienen lugar en centros hospitalarios y que además un alto porcentaje de defunciones neonatales ocurren en el primer día de vida, sería recomendable establecer un sistema de registro hospitalario tanto de nacimientos como de defunciones, lo que aseguraría una mejora sustancial en la cobertura del registro.

Los datos de esta investigación sugieren que esta recomendación es prioritaria para la Secretaría de Salud y para el isssTe, donde se encontraron los porcentajes más altos de subregistro. Este fenómeno seguramente se reproduce en otros estados.

En lo que compete a la Secretaría de Salud, es necesario que organice un sistema ágil y operativo que recabe y procese las copias de los certificados de defunción, pues es información que actualmente se desaprovecha y que constituiría una útil fuente de datos para el sector salud.

Un instrumento que ayudaría al estudio de la mortalidad perinatal sería la implantación de un certificado de defunción que haga posible el estudio conjunto tanto de muertes fetales tardías como de las neonatales tempranas, por lo que será necesario establecer normas para la captación de muertes fetales. Asimismo, en el caso de las muertes neonatales tempranas, es urgente unificar criterios con respecto a la definición de "nacido vivo" que maneja la Dirección General de Estadística (OMS, 1968), y la que se encuentra en el Código civil del Distrito Federal. De la misma ma- 
nera, es necesario unificar las definiciones de las distintas entidades del país, ya que también existen diferencias en sus respectivos códigos civiles en relación a la definición de "nacido vivo".

Es interesante destacar el hallazgo de no transferencia de muertes fetales a neonatales. Es posible que el fenómeno de transferencia se limite a nacimientos atendidos por personal no médico, por lo que su frecuencia debe ser mayor en otros estados y particularmente en el medio rural.

Otro factor muy relacionado con el subregistro es el peso al nacer de los recién nacidos, que ha sido considerado como un buen predictor de riesgo de muerte perinatal. Nuestros hallazgos coinciden con los de estudios como los de McCarthy y otros (1980) y Legarreta y otros (1973), en los que se obtuvieron altos porcentajes de subregistro en los recién nacidos menores de 1500 gramos.

Hay evidencias de que el bajo peso al nacer está condicionado de manera fundamental por una serie de factores biológicos y socioeconómicos de la madre. Entre los más importantes está la edad (madres muy jóvenes), la baja estatura y la escasa escolaridad materna, así como también el bajo nivel socioeconómico (Instituto de Desarrollo de la Salud, 1981).

Las características mencionadas coinciden con los resultados obtenidos en este estudio, en relación con ciertas variables socioeconómicas de las madres que no registraron sus muertes perinatales en el Registro Civil. Las mujeres más jóvenes con alta paridad y sujetas a condiciones laborales desfavorables, son las que subregistran más sus muertes perinatales. Aquí es importante resaltar la influencia que ejerce el estado civil en el subregistro. El subregistro fue mayor entre los hijos de madres separadas, viudas y solteras, algo que probablemente se deba a que la maternidad en estas condiciones representa una situación de mayor desventaja social para las mujeres por la marginación a que se ven sujetas; si agregamos el problema de la maternidad no deseada, es comprensible que al ocurrir el deceso del producto, las madres se sientan liberadas de una carga, con la consecuente reserva para acudir al Registro Civil.

En cuanto a la edad al morir, a pesar de las evidencias observadas en estudios como los de Ordorica y otros (1978) sobre un mayor subregistro en el primer día de vida, nuestros hallazgos no muestran una tendencia de este tipo, pues los porcentajes de subregistro en los primeros siete días de vida son muy similares entre sí. Esto quizás se deba a que a pesar de que la mortalidad es mayor en las primeras 24 horas de vida que en los días siguientes, la inscripción depende más de factores socioeconómicos que condicionan la asistencia de las madres al Registro Civil.

La certificación médica fue de $100 \%$, pero esta cifra no garantiza la buena calidad de la información, pues en muy pocos casos el médico que certificó fue el mismo que se encargó de atender al fallecido; puede esperarse, por tanto, que una gran proporción de los diagnósticos sobre las causas de la muerte estén basados en datos poco precisos. Asimismo, la 
calidad de la información de los certificados tiene deficiencias que van desde $7 \%$ de no concordancia para la edad al morir (porcentaje parecido al encontrado en el estudio de Varela y Narro (1981), hasta $45 \%$ de no concordancia para la edad de gestación.

\section{Conclusiones y recomendaciones}

Es conocida la utilidad de las cifras de mortalidad en la aplicación de programas de salud, por lo que una cobertura completa de defunciones, así como la buena calidad de la información, resultan de crucial importancia. Además, conocer algunos factores biológicos y socioeconómicos de riesgo de la madre ha mostrado ser de gran utilidad para la regionalización de los servicios médicos perinatales. Por lo anterior, es necesario establecer medidas tendentes a mejorar la organización y funcionamiento de los procesos involucrados en el registro de defunciones.

Debe señalarse que el dato de peso al nacer constituye una herramienta básica de análisis tanto de nacidos vivos como de defunciones perinatales. Se recomienda su incorporación a los certificados de defunción y a las actas de nacimiento. Un avance importante en este sentido se dio con la creación del nuevo certificado de defunción fetal por la Secretaría de Salud (Diario Oficial, 21 de noviembre de 1986), donde se solicita el peso al nacer para el registro de todas las defunciones fetales. Desgraciadamente este certificado no se acompaña de una redefinición de "muerte fetal", para que únicamente se captasen las que ocurren después de 19 semanas de gestación. La información de las que ocurren antes tiene poca utilidad. Esto se deriva del obvio subregistro de estas muertes, que en realidad son abortos, así como de las dificultades que existen para interpretar su significado desde el punto de vista de salud pública. Un aborto no necesariamente puede calificarse como un daño a la salud, ya que con frecuencia se recurre a él como método contraceptivo.

Es importante que las instituciones que proporcionan servicios de salud así como las encargadas de formar profesionales médicos, creen programas de adiestramiento en la elaboración de certificados de defunción, destacando la importancia del llenado correcto de estos documentos. Finalmente deben realizarse esfuerzos para superar los aspectos geográficos y económicos que limitan o impiden el acceso al Registro Civil y que por ende repercuten en la calidad de las estadísticas vitales. Éste parece ser el único camino para resolver el problema que enfrentó este trabajo por la falta de concordancia entre los datos contenidos en el certificado y los del cuestionario aplicado a las madres. 


\section{Bibliografía}

Aguirre, M.A. y C.S. Camposorterga (1980), "Evaluación de la información básica sobre mortalidad infantil en México", en Demografía y Economía, 44: 447.

Bobadilla, J.L. (1982), "La mortalidad en México", en F. Ortiz (comp.), Vida y muerte del mexicano, Folios Editores, México. (1985), Quality of. Perinatai Care in Mexico City, trabajo presentado al International Development Research Centre, México.

(1986), Quality of. Perinatai Medical Care in México City. An Epidemiological Study on the Effects of. Medical Care Quality on Perinatai Mortality, tesis doctoral presentada en la University of London.

Canales, M. y otros (1980), "El subregistro de la natalidad y la mortalidad en una comunidad rural en México", en Salud pública de México, xxII: 123.

Cordero, Eduardo (1968), "La subestimación de la mortalidad infantil en México", en Demografía y Economía, 11: 44.

Departamento del Distrito Federal (1980), Código civil para el Distrito Federal en materia común y para toda la República en materia federal, DDF, México.

Frost, F y otros (1982), "Completeness of Infant Death Registration for Very Low Birthweight Infant: Washington State 1978-79", en Public Health Briefs: 72, 740.

Instituto de Desarrollo de la Salud (1981), Investigación perinatai, Editorial Científica Técnica, La Habana.

Legarreta, RA. y otros (1973), "Omisión del registro de defunciones de niños ocurridas en maternidades. Santiago de Chile", en Bol. OPS, Lxxv: 308.

Me Carthy, B. y otros (1980), "The Underregistration of Neonatal Deaths: Georgia 1974-1977", en AJPH, 70: 977 .

Natali, Susana (1978), "Los sistemas de información de las estadísticas de natalidad y mortalidad en México", en Varios, Investigación Demográfica en México, Conacyt, México.

Ordorica, M.M. y otros (1975), "Evaluación de la mortalidad infantil en la República mexicana 1930-1970", en Evaluación y Análisis, III: 4. (1978), "El subregistro de la mortalidad infantil en 1976 (un estudio de campo)", en Evaluación y Análisis, III: 1.

Organización Mundial de la Salud (1968), Clasificación internacional de enfermedades, oMs, Ginebra.

Partida, Vi (1982), "Problemas en la estimación de la mortalidad a nivel regional en México: caso del Distrito Federal y el estado de México en 1970", en Varios, Investigación Demográfica en México 1980, Conacyt, México.

Rojas O. y T. Sánchez (1977), "Cobertura y calidad de la información estadística sobre la mortalidad perinatal en Cuba", en Bol. OPS, Lxxv1, 334.

Secretaría de Gobernación (1982), Diagnóstico del Registro Civil en México, 1980, Dirección General del Registro Nacional de Población e Identificación Personal, México.

Secretaría de Programación y Presupuesto (1982), Clasificación mexicana de ocupaciones, 1980, Coordinación General de los Servicios Nacionales de Estadística, Geografía e Informática, México, vol. 2.

Secretaría de Salubridad y Asistencia (1981), Seguimiento retrospectivo de defunciones ocurridas en 1978-79 (resultados básicos de la encuesta), Unidad de Información, México.

Suárez, Patricia (1981), Estimation of. Underregistration of. Infant Deaths in Mexico, tesis de maestría presentada en la London School of Higiene an Tropical Medicine, Centre for Population Studies, Londres.

Varela, L. y J.R. Narro (1981), "El registro de defunciones en una comunidad rural", en Salud pública de México, xxIII: 65. 
